\title{
The Diabetic Neuropathies: Practical and Rational Therapy
}

\author{
J. Robinson Singleton, M.D. ${ }^{1,2}$ A. Gordon Smith, M.D. ${ }^{1,3}$
}

1 University of Utah School of Medicine, Salt Lake City, Utah

${ }^{2}$ Department of Neurology

${ }^{3}$ Department of Neurology and Pathology

Address for correspondence and reprint requests A. Gordon Smith, MD, Department of Neurology and Pathology, University of Utah School of Medicine, 30 North 1900 East, SOM3R242, Salt Lake City, UT

Semin Neurol 2012;32:196-203. 84132 (e-mail: Gordon.smith@hsc.utah.edu).

\begin{abstract}
Keywords

- diabetes

- peripheral neuropathy

- diabetic amyotrophy

Diabetes is associated with a variety of chronic and acute neuropathies. In this article, the authors summarize the clinical features of the most common diabetic neuropathies, focusing on those for which therapy is available or under active investigation. Distal symmetric polyneuropathy (DSP) is the most common form. Potential treatments for DSP are discussed in four broad themes: (1) medication and lifestyle therapy to improve hyperglycemia, insulin resistance, and attendant features of metabolic syndrome, including obesity and dyslipidemia; (2) pharmacologic therapy to alter neuropathy natural history aimed at rational targets from known pathophysiology; (3) symptomatic relief of neuropathic pain; and (4) treatment to prevent complications of neuropathy, including stasis ulcers and falls. The approach to the most common acute diabetic neuropathies is also reviewed.
\end{abstract}

\section{Clinical Features of Diabetic Neuropathy}

Distal symmetric polyneuropathy (DSP) is the most common neuropathic complication of diabetes; $50 \%$ or more of patients develop DSP, and up to $20 \%$ have clinical features of neuropathy at the time of diabetes diagnosis. Distal symmetric polyneuropathy causes "positive" and "negative" sensory symptoms in a length-dependent pattern, affecting the toes and distal foot, spreading over time to the ankle and proximally. Patients may describe numbness, or may be unaware of their sensory loss until demonstrated on exam. Positive symptoms consist of abnormal spontaneous "paresthesias" such as pricking, tingling, or burning; "allodynia," perception of nonpainful stimuli as painful; or "hyperalgesia," inappropriate exaggeration of painful sensation. Two in five diabetic patients with DSP experience neuropathic pain, which is often the principal source of disability. The pain is often described as "deep aching," "burning," "electric," "tingling," or "sharp." Although pain frequently limits activities and is worse with walking, it is most severe at nighttime and sometimes inhibits sleep. Pain is typically moderate to severe. The average pain intensity in a survey of 105 painful DSP patients was 5.8/10. ${ }^{1}$

Distal symmetric polyneuropathy is a major cause of disability and reduced quality of life. Those with severe neuropathy may suffer painless injury. With more advanced disease, motor denervation can result in foot intrinsic muscle atrophy, exaggerated foot arch, and hammer toes. Foot and ankle instability due to weakness and reduced proprioception leads to falls, sprains, foot bone fractures, and other orthopedic injuries. Loss of protective sensation combined with altered sweating and poor wound healing predispose patients with DSP to foot ulcers, Charcot foot abnormalities, and amputation.

Evaluation should proceed from focused questions about pain, paresthesias, sensory loss, and weakness to an exam tailored to detection of length-dependent nerve injury. Strength is often normal, but there may be atrophy of foot muscles. Atrophy of hand muscles is more often due to focal compressive mononeuropathies of ulnar or median nerves. Deep tendon reflexes are characteristically reduced or absent at the ankles and well-preserved distal reflexes in the face of other neuropathic features should prompt consideration of a structural myelopathy or a dorsal column neuropathy, such as B12 deficiency. Sensory testing should evaluate the severity (absence vs reduced) and distribution of sensory loss to modalities associated with small unmyelinated (pain and autonomic) and large myelinated (proprioceptive, touch,
Issue Theme Neuromuscular Therapy from Bench to Bedside; Guest Editor, A. Gordon Smith, MD
Copyright @ 2012 by Thieme Medical Publishers, Inc., 333 Seventh Avenue, New York, NY 10001, USA. Tel: +1(212) 584-4662.
DOI http://dx.doi.org/ 10.1055/s-0032-1329195. ISSN 0271-8235. 
and motor) fibers. Pin sensation can be rapidly mapped to establish the proximal extent of sensory deficit. Timed vibration with a $128 \mathrm{~Hz}$ tuning fork provides a semiquantitative measure with a broad dynamic range. Nerve conduction studies and electromyography should be considered in patients who present with rapidly progressive symptoms or significant asymmetry or motor involvement.

Diabetic autonomic neuropathy is common, but underrecognized. Most patients with diabetic autonomic neuropathy do not have symptoms of a generalized dysautonomia. Instead, many are asymptomatic, or present with vague symptoms of dizziness, poor balance, nausea, abdominal pain, or sexual dysfunction. Thus, a high index of suspicion for diabetic autonomic neuropathy is required. Diabetic autonomic neuropathy significantly increases risk for cardiac death and causes disability due to orthostatic hypotension, syncope, erectile dysfunction, gastroparesis, diarrhea, and hypoglycemia. Coronary artery disease is often evaluated because it predicts greater all-cause mortality and can be detected using simple tests of vagal heart rate response to deep breathing or Valsalva. Erectile dysfunction is reported by $27 \%$ of men with newly diagnosed diabetes, and in 20 to $40 \%$ of men with metabolic syndrome and prediabetes. ${ }^{2}$ Sensitive tests for defects in peripheral sweat generation in response to acetylcholine find reduced responsiveness in a quarter of newly diagnosed diabetic patients.

\section{Focal, Acute, and Regional Forms of Diabetic Peripheral Nerve Injury}

Compressive mononeuropathies, especially ulnar, median, and peroneal, are more common in diabetes. Bilateral and nondominant median mononeuropathy at the wrist are more common in diabetes, ${ }^{3}$ although obesity, rather than diabetes, may be the primary driver of increased carpal tunnel syndrome risk. ${ }^{4}$ Hand numbness suggests bilateral median mononeuropathies at the wrist (carpal tunnel syndrome) and /or ulnar mononeuropathy at the elbow (cubital tunnel syndrome) in preference to advanced DSP. Nerve conduction studies should be performed to confirm compressive ulnar or median mononeuropathies, and to screen for associated axonal injury. Those with pure demyelinating injury may respond to nocturnal neutral wrist splints and a regimen of studious avoidance for positional cubital tunnel compression. Patients with active denervation should be referred for carpal tunnel decompression, which is nearly as effective for diabetes patients as for normoglycemic controls, improving digital sensation in two-thirds. ${ }^{5}$ Ulnar transposition from the cubital tunnel improves symptoms in only 50 to $60 \%$ of ulnar mononeuropathy patients.

Diabetes is associated with several acute neuropathic complications. Diabetic lumbosacral radiculoplexus neuropathy (DLRPN or "diabetic amyotrophy") typically presents with the abrupt onset of severe unilateral thigh pain. This is followed by progressive atrophy and weakness, involving proximal more than distal muscles. Patients may experience dramatic weight loss and many are rendered wheelchair dependent. ${ }^{6}$ Distal onset and contralateral limb involvement are common, and patients with severe upper-extremity symptoms or variant DLRPN with motor predominance, absence of pain, and greater clinical symmetry have been described, further broadening the spectrum of this disorder. ${ }^{7}$ Most patients experience progression over many months with subsequent improvement, but are left often with permanent deficits. ${ }^{6}$

Diabetic lumbosacral radiculoplexus neuropathy typically affects older patients with type 2 diabetes. Unlike DSP, DLRPN risk is not related to diabetic control or duration. Indeed, DLRPN patients typically have shorter diabetes duration than those with DSP. Electrodiagnostic studies demonstrate evidence of a polyradiculoneuropathy. Cerebrospinal fluid examination is characterized by elevated protein concentration without pleocytosis. Nerve biopsies demonstrate a microvasculitis. ${ }^{8}$ These features support an autoimmune etiology. A retrospective case series reported benefit for neuropathic pain in five DLRPN patients treated with intravenous immunoglobulin (IVIg), ${ }^{9}$ but a small randomized trial did not demonstrate significant disease modification with IV methylprednisolone. A recent Cochrane review concluded that definitive studies are lacking, ${ }^{10}$ perhaps setting the stage for a carefully designed randomized trial of IVIg in this disorder.

\section{Metabolic Syndrome Features and Their Role in Diabetic Neuropathy}

Glycemic control has long been the cornerstone of DSP therapy. However, large epidemiologic and treatment studies in patients with diabetes suggest obesity and dyslipidemia may be equally important risk factors. Hyperglycemia does not exist in isolation, and is one component of a broader metabolic syndrome with features initially identified due to their prediction for cardiovascular disease. ${ }^{11}$ Metabolic syndrome is defined as the presence of three of the following, increased fasting glucose, hypertriglyceridemia, decreased high-density lipoprotein-C (HDL-C), ethnicity-specific central obesity, and elevated blood pressure. Active drug treatment for any of these conditions in a given patient also meets criteria. $^{12}$

Several metabolic syndrome features have been linked to neuropathy, particularly obesity and dyslipidemia, independent of hyperglycemia. ${ }^{13}$ The largest studies have examined metabolic syndrome contribution to neuropathy risk in patients with known diabetes. The EuroDiab study found DSP in $28 \%$ of type 1 diabetic patients. It was noted that many patients with good glucose control (hemoglobin A1c $<7 \%$ ) had DSP, suggesting other risk factors were important. Among patients who did not have neuropathy at baseline, 23.5\% developed neuropathy after an average follow up of 7.3 years. Hypertension, serum lipids and triglycerides, body mass index (BMI), and smoking were each identified as independent risk factors. ${ }^{14,15}$ Other studies have noted higher obesity rates in patients with idiopathic neuropathy, both with and without abnormal glucose metabolism. ${ }^{13,16}$ In addition, there is also evidence that 
obesity is associated with abnormal autonomic function, early or subclinical autonomic neuropathy. ${ }^{17}$

Hypertriglyceridemia is significantly more common in those with idiopathic neuropathy, ${ }^{18}$ and may be involved in its development and progression. Among 28,700 diabetic patients, serum triglyceride level was an independent stepwise risk factor for lower extremity amputation, often a consequence of neuropathy. ${ }^{19}$ In a cohort study of patients with mild to moderate diabetic neuropathy, elevated triglycerides significantly correlated with loss of sural nerve myelinated fiber density over the study duration (52 weeks), independently of disease duration, age, or diabetes control. ${ }^{13,18,20}$

\section{Treatment of Hyperglycemia and Metabolic Syndrome}

Aggressive glycemic control has been shown in large, welldesigned clinical trials to reduce the neuropathy risk in type 1 diabetes. The Diabetes Control and Complications Trial (DCCT) randomized 1441 patients with type 1 diabetes to intensive or standard glucose control. During the long-term follow-up, glycemic control was similar between patients initially randomized to the different treatment groups. Despite this fact, those who had 5 years of intensive control during the DCCT were less likely to have neuropathy 14 years later than those who had standard therapy (25\% vs $35 \%$, $p<0.001) .{ }^{21}$ Similar data supporting a benefit for aggressive glycemic control in type 2 diabetes are lacking. The ACCORD study, which randomized 10,251 early diabetic subjects (HgbA1c of $>7.4 \%$ ) to either intensive (HgbA1c goal of $6.5 \%$ ) or standard (goal 7.0-7.9\%) therapy found no reduction in the risk of incident clinical neuropathy. ${ }^{22}$ A recent Cochrane Review of this topic concludes that aggressive glycemic control does not reduce clinical neuropathy risk. ${ }^{23}$

The Steno- 2 trial provides the best data on potential neuroprotective effects of metabolic syndrome control in patients with diabetes. Patients with type 2 diabetes were randomized to receive standard treatment or aggressive multimodal therapy to normalize weight, hypertension and lipid abnormalities, together with smoking cessation. Those in the multifactorial treatment arm were significantly less likely to develop coronary, cerebral, or peripheral vascular occlusive disease, but also nephropathy, retinopathy, and autonomic neuropathy. ${ }^{24}$ Somatic neuropathy and associated pain were not evaluated.

An evolving literature supports a role for exercise in neuropathy prevention and therapy. One prospective study compared neuropathy incidence in 78 type 2 diabetes patients randomized to supervised treadmill walking 4 hours weekly or bland counseling. ${ }^{25}$ HgbA1c did not improve significantly for either cohort, but exercise subjects improved fitness doubled $\mathrm{VO}_{2}$ max and reduced waist circumference. Clinical neuropathy developed in $30 \%$ of nonexercisers compared with $7 \%$ of exercisers ( $p<0.05$ ). A smaller unrandomized study in subjects with DSP found 10 weeks of supervised exercise significantly improved reported neuropathic pain, as well as cutaneous axon sprouting. ${ }^{26}$ These results suggest that exercise may impact neuropathy through a mechanism other than improvement in glucose control.

Generally, however, once DSP is established, it has proved remarkably resistant to treatment. No human clinical trial has demonstrated reversal or improvement of DSP even with complete resolution of diabetes. Patients with type 1 diabetes who underwent pancreatic transplant were compared with matched subjects who received best medical therapy. Compared with medical therapy subjects, who experienced unrelenting decline in clinical measures of peripheral nerve function, transplant recipients showed a stabilization of neuropathy progression, but minimal improvement over many years. ${ }^{27}$ With neuropathy progression, reversible metabolic factors tend to be supplanted by irreversible structural injury. ${ }^{28}$ Even in rodent models, spontaneous reversion to normoglycemia does not result in significant improvement of chronic diabetic neuropathy. ${ }^{29}$ These observations suggest future therapeutic efforts should focus on very early disease or even neuropathy prevention.

\section{Treatment Based on Recognized Steps in Diabetic Pathophysiology}

The pathophysiology of DSP is complex and several important mechanistic pathways have been identified. Neuropathy likely results as a combination of direct axonal injury due to metabolic consequences of hyperglycemia, insulin resistance, and toxic adiposity in addition to endothelial injury and microvascular dysfunction leading to nerve ischemia. Diabetes causes functional deficits in nitric-oxide-mediated microvascular reactivity as well as a structural microangiopathy that share pathologic features with microvascular injury to the retina. Additional relevant metabolic pathways include oxidative and nitrosative stress, ${ }^{30,31}$ accumulation of advanced glycation end products, ${ }^{32}$ direct toxic effects of free fatty acids and proinflammatory adipokines. ${ }^{33}$ These pathways produce microischemia of nutrient nerve arterioles, dysregulate axonal mitochondrial function, inhibit axonal transport of proteins necessary for distal axonal function, and elicit an autoimmune response. ${ }^{32}$

Pathogenic elucidation spawned a plethora of animal models and medications designed to block specific putative pathogenic pathways. Despite several promising therapies in cell culture and animal models of diabetic neuropathy, no rational treatment has clearly proven effective at reversing or slowing progression. Multiple trials of small vessel vasodilatory agents showed no clinical response, ${ }^{34}$ nor did a large trial of nerve growth factor (NGF). ${ }^{35}$ Trials of aldose reductase inhibitors (ARI), which control entry of excess glucose into the polyol pathway and reduce glucose mediated oxidative stress and microvascular disease in animals, have generally shown no efficacy in humans. ${ }^{36,37}$

Three related potential medications for DSP combine neuropathic pain relief with possible direct effect on neuropathic injury. Alpha lipoic acid, acetyl-L carnitine, and benfotiamine each act to reduce oxidative stress, which has been identified as a key component of neuropathy pathogenesis. In diabetes, excess lipids and glucose both produce oxidative 
free radicals that directly damage axonal mitochondria and divert nitric oxide from its normal vasodilatory role, resulting in impaired vasoregulation and ischemia of nutritive arterioles. ${ }^{38}$ Alpha lipoic acid (ALA) is an orally bioavailable antioxidant. The Symptomatic Diabetic Neuropathy (SYDNEY) Study randomized diabetic neuropathy subjects to double-blinded treatment with either placebo or up to $1800 \mathrm{mg}$ of oral ALA. Over the 5-week treatment course, subjects treated with any of the ALA doses reported a significantly greater reduction in neuropathic pain than did the placebo-treated controls. ${ }^{39}$ Most clinical measures of neuropathy severity did not significantly improve; previous studies had reported modest improvement in neuropathy measures following IV ALA injection. ${ }^{40}$ Alpha lipoic acid is regulated as a drug in many European nations, but is available in the United States as a dietary supplement. ALA may be started at a dose of $300 \mathrm{mg}$ by mouth daily and titrated as high as $600 \mathrm{mg}$ twice daily. ALA may lower blood glucose and thiamine stores, and has unpredictable effects on thyroid function.

Diabetes is associated with reduced serum levels and cellular concentrations of acetyl-L carnitine (ALC), another antioxidant that has been shown to inhibit lipid peroxidation and increase nitric oxide synthase and nitric oxide in experimental models. Two parallel randomized, blinded controlled trials used measures including the Visual Analog Pain Scale (VAS), and morphometric analysis of sural nerves to assess neuropathy severity at baseline and after 52 weeks of treatment; 1257 diabetic neuropathy subjects received either placebo or one of two ALC doses. ${ }^{41}$ ALC significantly improved sural morphology and VAS. These also improved significantly with the larger ALC dose. ALC is available as a nutritional supplement, often in combination with ALA, and a target dose of $500 \mathrm{mg}$ daily is best supported.

Benfotiamine or S-benzoylthiamine O-monophosphate is a vitamin B1 derivative with antioxidant properties. A phase III study randomized 165 diabetic neuropathy subjects to either placebo or benfotamine. ${ }^{42}$ Subjects receiving active drug showed significant improvement in the neuropathyspecific Total Symptom Score and its pain subscore over the 6week trial, with greater benefit in the $600 \mathrm{mg}$ dose group. Taken together, trials of these medications strongly implicate oxidative stress as a contributor to neuropathy pathogenesis.

Delivery of a putative therapeutic agent to the target organ (dorsal root ganglia or nerve) without limiting off-target side effects has proven challenging. This issue was particularly problematic in the phase III NGF trial, in which even a low dose of NGF was complicated by symptomatic hyperalgesia. ${ }^{43}$ One solution to this problem is use of delivery vectors derived from the herpes simplex virus, which has trophism for dorsal root ganglia neurons. A recent phase I study of a replication deficient HSV vector expressing the gene for preproenkephalin in patients with cancer-related pain demonstrated an apparent dose-related treatment effect. ${ }^{44}$ Other studies have used plasmids (naked DNA) containing growth factor genes to deliver therapy to nerve via intramuscular injection. A small trial of a plasmid containing the gene for vascular endothelial growth factor suggested benefit, and other trials using this delivery system are underway. ${ }^{45}$
Numerous physical, surgical, or energy therapies are purported to improve neuropathy by increasing vascular or microvascular blood flow. These include "triple decompressive surgery" to release the peroneal nerve at the fibular head and foot dorsum, and the tibial nerve at anatomic tunnels in the foot, ${ }^{46}$ fixed or pulsed magnetic field therapy, ${ }^{47,48}$ near infrared phototherapy, ${ }^{49}$ and various nonpenetrating laser therapies. In all cases, either rigorous blinded and randomized trials have not been performed, or as in the case of magnetic field therapy, these studies have not demonstrated improvement in nerve function. ${ }^{50}$

\section{Treatment of Neuropathic Pain}

A majority of DSP patients complain of neuropathic pain, making diabetes the single most common cause of neuropathy and neuropathic pain in industrialized countries. A crosssectional study comparing 350 people with diabetes to 344 age- and sex-matched controls in Liverpool found chronic painful neuropathy in $16 \%$ of diabetic patients, but only $4 \%$ of controls. Evaluation of the same cohorts 5 years later found neuropathic pain continued for $77 \%$ of diabetic subjects, without significant improvement as measured by the Visual Analog Pain Scale despite multimodal pain treatment. ${ }^{51}$ Onethird had not received specific treatment despite requesting pain medication from their physician. This result mirrors the frustrating experience of many patients and physicians in treating established neuropathic pain in DSP. ${ }^{52}$ A wide variety of antidepressants, antiepileptic drugs, opiates, and mixed serotonin/norepinephrine reuptake inhibitors have been shown to significantly reduce neuropathic pain compared with placebo in randomized controlled trials, ${ }^{52}$ but pain relief is incomplete for most patients.

Tricyclic antidepressants (amitriptyline, nortriptyline, desipramine) have been mainstays of neuropathic pain treatment, with efficacy proven in several well-designed studies. ${ }^{53}$ Tricyclic antidepressants are inexpensive, and because of their long half-life, dosing is simple. When administered 2 hours before bedtime, amitriptyline often aids in sleep initiation, even at low dose. This sedative effect is durable, and is of significant benefit for patients who report that neuropathic pain increases with rest, and makes it hard to fall asleep at night. A typical dose of amitriptyline is $50-200 \mathrm{mg}, 2$ hours before bedtime. To minimize side effects, patients should gradually increase their dose, beginning at $10 \mathrm{mg}$, and increasing by $10 \mathrm{mg}$ increments every 5 days to an initial plateau dose of $50 \mathrm{mg}$ nightly. Dosing increases can then proceed in $25 \mathrm{mg}$ increments. Because of the risk for partial or complete heart block, older patients should have an electrocardiogram in evaluation of conduction defects. Orthostatic hypotension, urinary hesitancy, fatigue, somnolence, and confusion are also common dose-related side effects. Patients taking monoamine oxidase inhibitors should not take tricyclics.

Gabapentin is structurally related to the pain-modulating neurotransmitter, g-aminobutyric acid (GABA), although it acts neither as a GABA agonist or antagonist. Its mechanism is thought to be related to binding to the $\alpha 2$ delta subunit of 
voltage gated $\mathrm{Ca}^{2+}$ channels. In several placebo controlled, randomized trials examining patients with painful neuropathies, gabapentin has been shown to significantly reduce neuropathic pain and improve sleep. ${ }^{54}$ In the largest clinical trials, doses in excess of $1800 \mathrm{mg}$ divided three times daily were often necessary for optimal pain relief. Dose escalation of gabapentin should begin at $300 \mathrm{mg}$ taken 2 hours before bedtime, increasing in $300 \mathrm{mg}$ increments every 3 to 7 days to $600 \mathrm{mg}$ three times daily. This conservative escalation schedule minimizes side effects. A therapeutic trial should involve at least 4 weeks at a therapeutic dose, with some patients requiring $4800 \mathrm{mg}$ daily in divided doses. Gabapentin is generally well tolerated, and renal excretion means there is no interaction with medications that undergo hepatic cytochrome p450 metabolism. Dizziness and somnolence are the only side effects reported to occur more frequently in patients taking gabapentin than in those receiving placebo.

Pregabalin $\left(\right.$ Lyrica $^{\circledR}$, Pfizer Pharmaceuticals, New York, NY) is structurally similar to gabapentin and offers a very similar pharmacokinetic, metabolic, and side-effect profile, with a somewhat faster onset of pain reduction. Pregabalin has been approved by the Food and Drug Administration (FDA) for treatment of neuropathic pain in diabetes, and for treatment of fibromyalgia. ${ }^{55}$ Dose escalation of pregabalin can begin at $75 \mathrm{mg}$ twice daily, and increase by $75 \mathrm{mg}$ increments to $150 \mathrm{mg}$ twice daily.

Duloxetine is a selective serotonin and norepinephrine reuptake inhibitor FDA-indicated for neuropathic pain associated with diabetic DSP, as well as for depression, anxiety, pain associated with fibromyalgia, and chronic musculoskeletal pain. In two randomized controlled trials, diabetic subjects with painful DSP of less than 6 months duration showed statistically better cumulative pain relief over the 12-week trial period when administered duloxetine $60 \mathrm{mg}$ daily compared with placebo. ${ }^{56}$ This is the recommended dose. Common side effects include nausea, somnolence, dry mouth, and constipation. Appetite suppression is common, but is often regarded as a benefit of the drug.

Gabapentin, pregabalin, and duloxetine are frequently offered in sequence to neuropathic pain patients, but have not been compared head-to-head in blinded or crossover trials. An open label trial in DSP patients who did not improve with gabapentin found no significant difference in efficacy between pregabalin and duloxetine. ${ }^{57}$ Retrospective review suggested a statistical advantage for pain relief to pregabalin (33\% responders) compared with duloxetine (21\%). ${ }^{58}$ Overall, none of these medications provides effective neuropathic pain control for the majority of DSP patients, and combination therapy has not been well evaluated.

Opiates have a defined role as adjunct treatment in poorly controlled neuropathic pain, but are often overlooked because of concerns for tolerance and addiction. ${ }^{59}$ The oral opiate oxycodone improved pain and quality of life in a small diabetic neuropathy cohort, ${ }^{60}$ and a larger study (338 subjects) found significantly enhanced pain relief when added in a randomized and double-blinded fashion to optimized gabapentin dosing. ${ }^{61}$ However, in a recent study of 62 diabetic neuropathy subjects, randomized addition of low-dose oxy- codone did not significantly improve the neuropathic pain effects of pregabalin. ${ }^{62}$ The novel mu-opioid receptor binding agent tapentadol has recently been shown superior to placebo in reducing neuropathic pain in a randomized trial cohort of 395 subjects with diabetic neuropathy. ${ }^{63}$

In practice, a short-acting agent, such as hydrocodone or oxycodone, can be used to slowly titrate the dose to efficacy. Once an effective dose has been found, conversion to a longacting ("time contingent") opiate such as extended release morphine (MS Contin) or oxycodone helps provide sustained pain relief with fewer side effects. A short-acting agent may be used for breakthrough pain or exacerbations. Ethanol and benzodiazepines synergize with opiates to cause respiratory depression and sedation. Patients should be counseled to avoid alcohol consumption. Constipation is the most common side effect of low-dose oral opiate use. Prophylactic institution of a bowel control regimen helps prevent constipation. Sedation and central respiratory depression are of concern at higher doses, and may be more dangerous in obese patients with obstructive sleep apnea.

Treating physicians are often reluctant to prescribe opiates due to concern for risk of addiction. Although physical dependence occurs in many patients, it is rarely a problem. A prospective study in patients with diabetes suggests that psychological dependence and addiction are rare when these agents are used for pain, even for long periods. ${ }^{64}$ Patients should taper off of opiates rather than stopping them suddenly. Patients at higher risk of addiction, such as those with a history of drug or alcohol abuse, may require referral to a pain specialist.

Tramadol, an agent that binds to opiate receptors and blocks reuptake of serotonin and norepinephrine, was significantly more effective than placebo in treating neuropathic pain in a 42-day double-blind placebo controlled study of 131 diabetic subjects, ${ }^{65}$ and in combination with acetaminophen was shown to be significantly more effective than placebo alone in a cohort of 160 subjects. ${ }^{66}$ Long-term use of Tramadol in patients with diabetic neuropathy did not result in significant tolerance or dose escalation. ${ }^{64}$

Mexiletine is an orally active local anesthetic agent that showed statistically significant reduction in Visual Analog Pain Scale pain ratings for diabetic neuropathy patients in two small trials at doses of 225-675 mg day. Nausea and other gastrointestinal complaints were the most common side effects. ${ }^{67}$

Capsaicin cream is applied topically, and acts to reduce pain sensation by depleting substance $P$ from proximal terminals of cutaneous nociceptive c-fibers. Its use is well validated in patients with herpes zoster. In a study of 13 patients with painful diabetic neuropathy, capsaicin reduced Visual Analog Pain Scale ratings while improving heat pain perception threshold. ${ }^{68}$ Capsaicin is practical only for patients with small areas of neuropathic pain. Pragmatically, few patients tolerate capsaicin therapy: the cream must be applied three to four times daily using rubber gloves to avoid affecting nonpainful skin or mucosa. Failure to maintain treatment for even one dose allows substance-P regeneration and recrudescence of pain. 
Several other anticonvulsants and antidepressants have been studied with mixed results. There are also several innovative and promising pain therapies in development (including the gene therapy approaches described above). The interested reader is referred to the review of neuropathic pain in this issue of Seminars in Neurology.

\section{Prevention of Neuropathy Complications: Falls and Ulcers}

Patients with peripheral neuropathy are at higher risk of falling, and this problem may be particularly acute in diabetes. Among 60 diabetic patients over the age of 55 years old, over one-third had fallen in the prior year. Neuropathy, sensory loss, and distal weakness were major risk factors. ${ }^{69}$ Bedside gait examination may be a relatively insensitive predictor of fall risk. Patients with neuropathy often fall when walking on uneven or irregular surfaces, and formal gait evaluation should include these testing conditions. ${ }^{70}$ Any DSP patient, or patients with poor lower extremity strength or vibration perception should be counseled regarding fall risk and consideration given to possible gait evaluation and physical therapy intervention.

Foot ulceration and consequent digit, foot, or limb amputation remains a common diabetic complication. Among 248 diabetic patients followed prospectively in tertiary care podiatric clinic, foot ulcers developed in $29 \%$ of subjects and $19 \%$ of feet over a mean 30-month follow-up. ${ }^{71}$ DSP increases the risk of ulceration sevenfold and contributes to over $60 \%$ of lower extremity amputations in diabetic patients. ${ }^{72}$ The increased risk is due to a combination of lack of protective sensation, abnormalities in blood flow that are often compounded by peripheral artery disease, abnormal sweating, and poor wound healing. ${ }^{73}$ Ulceration and amputation risk is also related to duration of neuropathy and severity of hyperglycemia. Daily self-examination, with a foot mirror if necessary; podiatric consultation and maintenance for toenails and bunions; orthotic foot support, and use of protective, widebased shoes with adequate toe box and ankle support are recommended for patients at risk for ulcers. If stasis ulcers develop, nonsurgical debridement, application of hydrogels, and empiric antibiotic coverage for wound flora are appropriate therapy. ${ }^{74}$

\section{Summary}

In summary, effective management of hyperglycemia, symptom control, and prevention of ulcers and infection through screening and surveillance remain mainstays of diabetic neuropathy management four decades after their introduction. Rational therapies based on evolving understanding of diabetic neuropathy pathogenesis have been largely unsuccessful in humans. This failure may reflect, in part, the advanced neuropathy present in subjects of previous human trials. More sensitive and quantitative measures of early peripheral nerve injury, including skin biopsy for intraepidermal nerve fiber density, and confocal corneal microscopy, hold promise to identify neuropathy patients early in their disease course, when rational treatments may be more effective. At the same time, traditional and rational diabetic neuropathy treatments will be supplemented by novel cellbased therapy and targeted drug delivery systems.

\section{References}

1 Galer BS, Gianas A, Jensen MP. Painful diabetic polyneuropathy: epidemiology, pain description, and quality of life. Diabetes Res Clin Pract 2000;47(2):123-128

2 Sairam K, Kulinskaya E, Boustead GB, Hanbury DC, McNicholas TA. Prevalence of undiagnosed diabetes mellitus in male erectile dysfunction. BJU Int 2001;88(1):68-71

3 Zambelis T, Tsivgoulis G, Karandreas N. Carpal tunnel syndrome: associations between risk factors and laterality. Eur Neurol 2010;63(1):43-47

4 Becker J, Nora DB, Gomes I, et al. An evaluation of gender, obesity, age and diabetes mellitus as risk factors for carpal tunnel syndrome. Clin Neurophysiol 2002;113(9):1429-1434

5 Thomsen NO, Cederlund R, Rosén I, Björk J, Dahlin LB. Clinical outcomes of surgical release among diabetic patients with carpal tunnel syndrome: prospective follow-up with matched controls. J Hand Surg Am 2009;34(7):1177-1187

6 Barohn RJ, Sahenk Z, Warmolts JR, Mendell JR. The Bruns-Garland syndrome (diabetic amyotrophy). Revisited 100 years later. Arch Neurol 1991;48(11):1130-1135

7 Garces-Sanchez M, Laughlin RS, Dyck PJ, Engelstad JK, Norell JE, Dyck PJ. Painless diabetic motor neuropathy: a variant of diabetic lumbosacral radiculoplexus Neuropathy? Ann Neurol 2011;69(6): 1043-1054

8 Tracy JA, Engelstad JK, Dyck PJ. Microvasculitis in diabetic lumbosacral radiculoplexus neuropathy. J Clin Neuromuscul Dis 2009;11 (1):44-48

9 Tamburin S, Zanette G. Intravenous immunoglobulin for the treatment of diabetic lumbosacral radiculoplexus neuropathy. Pain Med 2009;10(8):1476-1480

10 Chan YC, Lo YL, Chan ES. Immunotherapy for diabetic amyotrophy. Cochrane Database Syst Rev 2009;(3):CD006521

11 Tesfaye S, Chaturvedi N, Eaton SE, et al; EURODIAB Prospective Complications Study Group. Vascular risk factors and diabetic neuropathy. N Engl J Med 2005;352(4):341-350

12 Alberti KG, Eckel RH, Grundy SM, et al; International Diabetes Federation Task Force on Epidemiology and Prevention; National Heart, Lung, and Blood Institute; American Heart Association; World Heart Federation ; International Atherosclerosis Society; International Association for the Study of Obesity. Harmonizing the metabolic syndrome: a joint interim statement of the International Diabetes Federation Task Force on Epidemiology and Prevention; National Heart, Lung, and Blood Institute; American Heart Association; World Heart Federation; International Atherosclerosis Society; and International Association for the Study of Obesity. Circulation 2009;120(16):1640-1645

13 Smith AG, Rose K, Singleton JR. Idiopathic neuropathy patients are at high risk for metabolic syndrome. J Neurol Sci 2008;273(1-2): $25-28$

14 Tesfaye S, Selvarajah D. The Eurodiab study: what has this taught us about diabetic peripheral neuropathy? Curr Diab Rep 2009;9 (6):432-434

15 Costa LA, Canani LH, Lisbôa HR, Tres GS, Gross JL. Aggregation of features of the metabolic syndrome is associated with increased prevalence of chronic complications in Type 2 diabetes. Diabet Med 2004;21(3):252-255

16 Singleton JR, Smith AG, Bromberg MB. Painful sensory polyneuropathy associated with impaired glucose tolerance. Muscle Nerve 2001;24(9):1225-1228 
17 Skrapari I, Tentolouris N, Perrea D, Bakoyiannis C, Papazafiropoulou A, Katsilambros N. Baroreflex sensitivity in obesity: relationship with cardiac autonomic nervous system activity. Obesity (Silver Spring) 2007;15(7):1685-1693

18 Hughes RA, Umapathi T, Gray IA, et al. A controlled investigation of the cause of chronic idiopathic axonal polyneuropathy. Brain 2004;127(Pt 8):1723-1730

19 Callaghan BC, Feldman E, Liu J, et al. Triglycerides and amputation risk in patients with diabetes: ten-year follow-up in the DISTANCE study. Diabetes Care 2011;34(3):635-640

20 Wiggin TD, Sullivan KA, Pop-Busui R, Amato A, Sima AA, Feldman EL. Elevated triglycerides correlate with progression of diabetic neuropathy. Diabetes 2009;58(7):1634-1640

21 Albers JW, Herman WH, Pop-Busui R, et al; Diabetes Control and Complications Trial /Epidemiology of Diabetes Interventions and Complications Research Group. Effect of prior intensive insulin treatment during the Diabetes Control and Complications Trial (DCCT) on peripheral neuropathy in type 1 diabetes during the Epidemiology of Diabetes Interventions and Complications (EDIC) Study. Diabetes Care 2010;33(5):1090-1096

22 Ismail-Beigi F, Craven T, Banerji MA, et al; ACCORD trial group. Effect of intensive treatment of hyperglycaemia on microvascular outcomes in type 2 diabetes: an analysis of the ACCORD randomised trial. Lancet 2010;376(9739):419-430

23 Callaghan BC, Little AA, Feldman EL, Hughes RA. Enhanced glucose control for preventing and treating diabetic neuropathy. Cochrane Database Syst Rev 2012;6:CD007543

24 Gaede P, Vedel P, Larsen N, Jensen GV, Parving HH, Pedersen O. Multifactorial intervention and cardiovascular disease in patients with type 2 diabetes. N Engl J Med 2003;348(5): 383-393

25 Balducci S, Iacobellis G, Parisi L, et al. Exercise training can modify the natural history of diabetic peripheral neuropathy. J Diabetes Complications 2006;20(4):216-223

26 Kluding PM, Pasnoor M, Singh R, et al. The effect of exercise on neuropathic symptoms, nerve function, and cutaneous innervation in people with diabetic peripheral neuropathy. J Diabetes Complications 2012; Epub ahead of print

27 Navarro X, Sutherland DE, Kennedy WR. Long-term effects of pancreatic transplantation on diabetic neuropathy. Ann Neurol 1997;42(5):727-736

28 Pfeifer MA, Schumer MP. Clinical trials of diabetic neuropathy: past, present, and future. Diabetes 1995;44(12):1355-1361

29 Kennedy JM, Zochodne DW. Experimental diabetic neuropathy with spontaneous recovery: is there irreparable damage? Diabetes 2005;54(3):830-837

30 Russell JW, Berent-Spillson A, Vincent AM, Freimann CL, Sullivan KA, Feldman EL. Oxidative injury and neuropathy in diabetes and impaired glucose tolerance. Neurobiol Dis 2008;30(3): 420-429

31 Obrosova IG, Drel VR, Oltman CL, et al. Role of nitrosative stress in early neuropathy and vascular dysfunction in streptozotocindiabetic rats. Am J Physiol Endocrinol Metab 2007;293(6): E1645-E1655

32 Sugimoto K, Yasujima M, Yagihashi S. Role of advanced glycation end products in diabetic neuropathy. Curr Pharm Des 2008;14 (10):953-961

33 Vincent AM, Hayes JM, McLean LL, Vivekanandan-Giri A, Pennathur S, Feldman EL. Dyslipidemia-induced neuropathy in mice: the role of oxLDL/LOX-1. Diabetes 2009;58(10):2376-2385

34 Coppey LJ, Davidson EP, Rinehart TW, et al. ACE inhibitor or angiotensin II receptor antagonist attenuates diabetic neuropathy in streptozotocin-induced diabetic rats. Diabetes 2006;55(2): 341-348

35 Apfel SC, Schwartz S, Adornato BT, et al. Efficacy and safety of recombinant human nerve growth factor in patients with diabetic polyneuropathy: a randomized controlled trial. rhNGF Clinical Investigator Group. JAMA 2000;284(17):2215-2221
36 Chalk C, Benstead TJ, Moore F. Aldose reductase inhibitors for the treatment of diabetic polyneuropathy. Cochrane Database Syst Rev 2007;(4):CD004572

37 Hotta N, Akanuma Y, Kawamori R, et al. Long-term clinical effects of epalrestat, an aldose reductase inhibitor, on diabetic peripheral neuropathy: the 3-year, multicenter, comparative Aldose Reductase Inhibitor-Diabetes Complications Trial. Diabetes Care 2006;29 (7):1538-1544

38 Singleton JR, Smith AG, Russell JW, Feldman EL. Microvascular complications of impaired glucose tolerance. Diabetes 2003;52 (12):2867-2873

39 Ziegler D, Ametov A, Barinov A, et al. Oral treatment with alphalipoic acid improves symptomatic diabetic polyneuropathy: the SYDNEY 2 trial. Diabetes Care 2006;29(11):2365-2370

40 Ziegler D, Nowak H, Kempler P, Vargha P, Low PA. Treatment of symptomatic diabetic polyneuropathy with the antioxidant alphalipoic acid: a meta-analysis. Diabet Med 2004;21(2):114-121

41 Sima AA, Calvani M, Mehra M, Amato A; Acetyl-L-Carnitine Study Group. Acetyl-L-carnitine improves pain, nerve regeneration, and vibratory perception in patients with chronic diabetic neuropathy: an analysis of two randomized placebo-controlled trials. Diabetes Care 2005;28(1):89-94

42 Stracke H, Gaus W, Achenbach U, Federlin K, Bretzel RG. Benfotiamine in diabetic polyneuropathy (BENDIP): results of a randomised, double blind, placebo-controlled clinical study. Exp Clin Endocrinol Diabetes 2008;116(10):600-605

43 Apfel SC, Schwartz S, Adornato BT, et al. Efficacy and safety of recombinant human nerve growth factor in patients with diabetic polyneuropathy: A randomized controlled trial. rhNGF Clinical Investigator Group. JAMA 2000;284(17):2215-2221

44 Fink DJ, Wechuck J, Mata M, et al. Gene therapy for pain: results of a phase I clinical trial. Ann Neurol 2011;70(2):207-212

45 Ropper AH, Gorson KC, Gooch CL, et al. Vascular endothelial growth factor gene transfer for diabetic polyneuropathy: a randomized, double-blinded trial. Ann Neurol 2009;65(4):386-393

46 Dellon AL. Neurosurgical prevention of ulceration and amputation by decompression of lower extremity peripheral nerves in diabetic neuropathy: update 2006. Acta Neurochir Suppl (Wien) 2007; $100: 149-151$

47 Weintraub MI, Wolfe GI, Barohn RA, et al; Magnetic Research Group. Static magnetic field therapy for symptomatic diabetic neuropathy: a randomized, double-blind, placebo-controlled trial. Arch Phys Med Rehabil 2003;84(5):736-746

48 Pieber K, Herceg M, Paternostro-Sluga T. Electrotherapy for the treatment of painful diabetic peripheral neuropathy: a review. J Rehabil Med 2010;42(4):289-295

49 Powell MW, Carnegie DH, Burke TJ. Reversal of diabetic peripheral neuropathy with phototherapy (MIRE) decreases falls and the fear of falling and improves activities of daily living in seniors. Age Ageing 2006;35(1):11-16

50 Chaudhry V, Russell J, Belzberg A. Decompressive surgery of lower limbs for symmetrical diabetic peripheral neuropathy. Cochrane Database Syst Rev 2008;(3):CD006152

51 Daousi C, Benbow SJ, Woodward A, MacFarlane IA. The natural history of chronic painful peripheral neuropathy in a community diabetes population. Diabet Med 2006;23(9):1021-1024

52 Ziegler D. Treatment of diabetic neuropathy and neuropathic pain: how far have we come? Diabetes Care 2008;31(Suppl 2): S255-S261

53 Max MB, Lynch SA, Muir J, Shoaf SE, Smoller B, Dubner R. Effects of desipramine, amitriptyline, and fluoxetine on pain in diabetic neuropathy. N Engl J Med 1992;326(19):1250-1256

54 Backonja M, Beydoun A, Edwards KR, et al. Gabapentin for the symptomatic treatment of painful neuropathy in patients with diabetes mellitus: a randomized controlled trial. JAMA 1998;280 (21):1831-1836

55 Arezzo JC, Rosenstock J, Lamoreaux L, Pauer L. Efficacy and safety of pregabalin $600 \mathrm{mg} / \mathrm{d}$ for treating painful diabetic peripheral 
neuropathy: a double-blind placebo-controlled trial. BMC Neurol 2008;8:33

56 Goldstein DJ, Lu Y, Detke MJ, Lee TC, Iyengar S. Duloxetine vs. placebo in patients with painful diabetic neuropathy. Pain 2005;116(1-2):109-118

57 Tanenberg RJ, Irving GA, Risser RC, et al. Duloxetine, pregabalin, and duloxetine plus gabapentin for diabetic peripheral neuropathic pain management in patients with inadequate pain response to gabapentin: an open-label, randomized, noninferiority comparison. Mayo Clin Proc 2011;86(7):615-626

58 Mittal M, Pasnoor M, Mummaneni RB, et al. Retrospective chart review of duloxetine and pregabalin in the treatment of painful neuropathy. Int J Neurosci 2011;121(9):521-527

59 Watson CP. The treatment of neuropathic pain: antidepressants and opioids. Clin J Pain 2000;16(2, Suppl):S49-S55

60 Watson CP, Moulin D, Watt-Watson J, Gordon A, Eisenhoffer J. Controlled-release oxycodone relieves neuropathic pain: a randomized controlled trial in painful diabetic neuropathy. Pain 2003;105(1-2):71-78

61 Hanna M, O'Brien C, Wilson MC. Prolonged-release oxycodone enhances the effects of existing gabapentin therapy in painful diabetic neuropathy patients. Eur J Pain 2008;12(6):804-813

62 Zin CS, Nissen LM, O'Callaghan JP, Duffull SB, Smith MT, Moore BJ. A randomized, controlled trial of oxycodone versus placebo in patients with postherpetic neuralgia and painful diabetic neuropathy treated with pregabalin. J Pain 2010;11(5):462-471

63 Schwartz S, Etropolski M, Shapiro DY, et al. Safety and efficacy of tapentadol ER in patients with painful diabetic peripheral neuropathy: results of a randomized-withdrawal, placebo-controlled trial. Curr Med Res Opin 2011;27(1):151-162

64 Harati Y, Gooch C, Swenson M, et al. Maintenance of the long-term effectiveness of tramadol in treatment of the pain of diabetic neuropathy. J Diabetes Complications 2000;14(2):65-70
65 Harati Y, Gooch C, Swenson M, et al. Double-blind randomized trial of tramadol for the treatment of the pain of diabetic neuropathy. Neurology 1998;50(6):1842-1846

66 Freeman R, Raskin P, Hewitt DJ, et al; CAPSS-237 Study Group. Randomized study of tramadol/acetaminophen versus placebo in painful diabetic peripheral neuropathy. Curr Med Res Opin 2007;23(1):147-161

67 Jarvis B, Coukell AJ. Mexiletine. A review of its therapeutic use in painful diabetic neuropathy. Drugs 1998;56(4):691-707

68 Forst T, Pohlmann T, Kunt T, et al. The influence of local capsaicin treatment on small nerve fibre function and neurovascular control in symptomatic diabetic neuropathy. Acta Diabetol 2002;39(1): $1-6$

69 Macgilchrist C, Paul L, Ellis BM, Howe TE, Kennon B, Godwin J. Lower-limb risk factors for falls in people with diabetes mellitus. Diabet Med 2010;27(2):162-168

70 DeMott TK, Richardson JK, Thies SB, Ashton-Miller JA. Falls and gait characteristics among older persons with peripheral neuropathy. Am J Phys Med Rehabil 2007;86(2):125-132

71 Pham H, Armstrong DG, Harvey C, Harkless LB, Giurini JM, Veves A. Screening techniques to identify people at high risk for diabetic foot ulceration: a prospective multicenter trial. Diabetes Care 2000;23(5):606-611

72 Ramsey SD, Newton K, Blough D, et al. Incidence, outcomes, and cost of foot ulcers in patients with diabetes. Diabetes Care 1999;22 (3):382-387

73 Gregg EW, Sorlie P, Paulose-Ram R, et al; 1999-2000 national health and nutrition examination survey. Prevalence of lowerextremity disease in the US adult population $>=40$ years of age with and without diabetes: 1999-2000 national health and nutrition examination survey. Diabetes Care 2004;27(7):1591-1597

74 Ndip A, Ebah L, Mbako A. Neuropathic diabetic foot ulcers evidence-to-practice. Int J Gen Med 2012;5:129-134 